\title{
Consumer Behavior Choice in the Era of Shared Mobility: The Role of Proximity, Competition, and Quality
}

\author{
Zhongju Zhang \\ W. P. Carey School of Business \\ Arizona State University \\ Tempe, Arizona 85287 \\ Zhongju.Zhang@asu.edu
}

\author{
Daoqin Tong \\ School of Geographical Sciences and \\ Urban Planning \\ Arizona State University \\ Tempe, Arizona 85287 \\ Daoqin.Tong@asu.edu
}

\author{
Wencong Cui \\ College of Geosciences \\ Texas A\&M University \\ College Station, Texas 77843 \\ ccuiww@tamu.edu
}

\begin{abstract}
Shared mobility services, which allow users to make point-to-point trips on an as-needed basis, have drastically impacted people's travel behavior in the last few years. In this study, we propose a decision choice model to examine the factors that influence the restaurant choice of individuals who use shared mobility services. Our model incorporates key elements from the spatial interaction model and the theory of the individual decision making from economics. We analyze individuals' travel behavior using trip-level data, along with point of interest data, restaurant reviews and average prices, and travel route characteristics. We find that the effect of proximity of a restaurant depends on the total distance of the trip. For shorter trips, an individual is less likely to choose a restaurant that is further away. However, if an individual decides to travel a long distance to a restaurant, she is more likely to choose a restaurant that is further. Additionally, with increasing travel distance (or competition) there is a decreased preference for a restaurant with a higher price. The quality (online reviews) of a restaurant does not seem to have a significant impact on the choice of the restaurant. Implications of the study are discussed.
\end{abstract}

\section{Introduction}

Shared mobility has become a global phenomenon in the transportation industry over the last few years. It broadly refers to the shared use of any transportation means such as a vehicle, bike, scooter, etc. (Shaheen et al., 2015). This concept originated in Europe where the first car sharing program (Shaheen et al., 1998) was launched in 1948 in Zurich, Switzerland, and the first shared bike system was established in 1965 in Amsterdam, Netherlands. Since then, shared mobility systems have expanded rapidly all over the world over the past decades. Recent advancements in mobile technologies and digital platforms have further pushed the frontier of this phenomenon. Among the shared mobility applications, dockless bike-sharing service suddenly became popular in 2016 (Qi et al., 2018). A few companies (such as Lime, Mobike, Spin, Uber) have recently started operating in many cities across the United States. Unlike traditional dock-based bike rent/share programs, services offered by these companies allow a customer to use a smart phone app to locate and unlock a bike nearby and ride it to the destination where he/she parks and locks the bike, making the bike available for other customers to use. The adoption of dockless bike-sharing service is rapid. Anecdotal evidence shows that dockless bike trips account for about $6.8 \%$ of all modal trips in China (Cui, 2018). City of Scottsdale, Arizona, reported 55,000 dockless bike rides in its first two months of operation; the average travel distance per trip is 1.35 miles with an average travel time of approximately 10 minutes.

On-demand shared mobility service offers a flexible, low-cost, and alternative mode of transportation, which can drastically impact people's mobility and travel behavior. For example, using dockless bike share, people have the opportunity to visit businesses and amenities that otherwise would not be possible by walking. It also extends the distance and the reach of public transit by providing an effective "last-mile" solution to the connectivity problem in cities. Some users have also reported that they drive less by using bike share, which could potentially reduce traffic congestion and parking space use. While shared mobility service has the potential to improve the efficiency of short-distance urban travel and create a positive impact on the community and environment, there is little research on how it impacts user's trip behavior with such choice.

In this paper, we propose a decision choice model to examine why an individual user of shared mobility service, starting from a location, chooses to travel to a 
particular restaurant (as opposed to other alternatives in close proximity of the origin). Figure 1 illustrates an example of a real trip and restaurant choice made by an individual. In the figure, the green dotted $\mathrm{O}$ represents the starting location (origin) of the individual. The red dotted D represents the destination restaurant that was chosen. The blue route represents the shortest bike route of the trip. D1, D2, and D3 are alternative restaurant choices. For each restaurant, the numbers in each black rectangle record the average price per person, the quality measures, and the distance (in meters) between the origin and that restaurant. In order to examine the effects as well as the interaction of those factors, this study combines the key elements from the spatial interaction model and the theory of the individual decision making from economics. We analyze the individual's travel behavior using trip-level data from dockless bike sharing in Shanghai, along with point of interest data, restaurant reviews and average prices, and travel route characteristics.

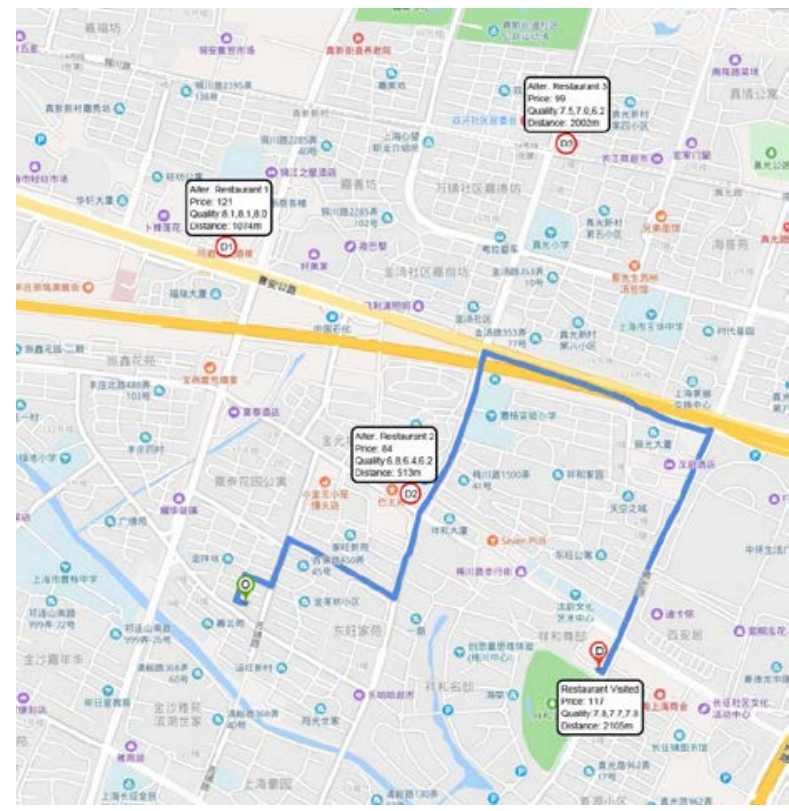

Figure 1: Illustration of an Individual Trip with Decision Choice (O: Origin, D: Destination, D1, D2, D3: Alternative Restaurants, Blue Line: Shortest Route from $\mathrm{O}$ to D)

We find that the effect of proximity of a restaurant depends on the total distance of the trip. For shorter trips, an individual is less likely to choose a restaurant that is further away. However, if an individual decides to travel a long distance to a restaurant, she is more likely to choose a restaurant that is further away. Additionally, with increasing travel distance (or competition) there is a decreased preference for a restaurant with a higher price. The quality (online reviews) of a restaurant does not seem to have a significant impact on the choice of the restaurant.

The rest of the paper is organized as follows. In Section 2, we discuss the theoretical foundations and formulate our hypotheses. Section 3 describes our data and empirical models. Section 4 presents the model results and discusses the findings. Section 5 offers concluding remarks.

\section{Theoretical Foundation and Hypotheses}

\subsection{Theory of Individual Decision Making}

Individual decision making is a rich topic and has been studied by various disciplines to analyze the choices and behaviors of individual agents. One of the classical approaches (Mas-Colell et al., 1995) to model individual choice behavior is the preference-based approach, which characterizes a decision maker's preference over a set of possible choices with certain rationality axioms and analyzes the consequences of these preferences for her choice behavior. In this setting, economists (e.g., Simon, 1955; Mas-Colell et al., 1995) typically develop models to study the decision choices of individual as a utility maximization problem subject to certain constraints (e.g., physical and budget constraints). The utility function is usually defined to represent the individual preference ordering over a set of possible choices. Depending on the context and the problem at hand, various specifications of the utility function have been proposed in the literature. Economists generally believe that the price of a product, the quality of the product, the price and quality of related products (competition) are major factors affecting the individual utility and the decisionmaking process. Other things being equal, consumers are more willing to choose product with better quality, lower price, or a combination of the two.

Consumer behavior models are another approach to study individual purchase decisions. These models usually combine both economic and psychological models, and are used by marketing professionals to understand how various attributes affect buying behavior. Marketing researchers (e.g., Kotler 2000) introduced a five-stage model to describe the consumer buying process: problem recognition, information search, evaluation of alternatives, purchase decision, and post purchase behavior. At each stage, important factors that may affect buyer behavior are identified. Sprotles and Kendall (1986) developed a consumer style inventory model and identified several distinct decision-making styles including quality conscious, 
brand conscious, price conscious consumers. Based on the above discussions, we hypothesize that:

H1: An individual is more likely to choose a restaurant with a higher quality (or lower price).

\subsection{Spatial Interaction Model}

Spatial interaction involves flow of people, goods, resources or information from one location to another (Smith, 1975). It covers a range of movement including daily commuting, retail store visits, and migration. A set of classic models have been developed to understand and quantify spatial interaction among places. Drawing on Newton's Law of Gravitation, Wilson (1971) developed gravity models and argued that spatial interaction between two places is proportional to their attractiveness and decays with distance. Gravity models have been applied and extended to study a range of spatial interaction, including retail shopping. Another theoretical framework for modeling spatial interaction is based on Stouffer's (1940) intervening opportunities model, which states that the amount of people going to a place is proportional to the number of opportunities at the place and inversely proportional to the number of intervening opportunities that exist between an origin and the place.

In addition to opportunities that exist between an origin and destination, Fotheringham (1983) introduced the notion of competing destinations. When a destination is surrounded by multiple destinations of a similar type, due to competition, the probability that the spatial interaction between an origin and that destination decreases. Spatial competition has been tested in retail markets (Davis 2006), popularity of tourism destinations (Hanink and Stutts 2002), and communication markets (Guldman 1999). Therefore, we hypothesize:

H2: An individual is more likely to choose a restaurant if the number of the alternative restaurants (competition) decreases.

H3: An individual is more likely to choose a restaurant that is closer in proximity.

It is worth to note that the spatial interaction models and the theories of individual decision-making focus on different aspects to model the decision choice problem of consumers, i.e., the restaurant choice of travelers. The former focus primarily on the spatial arrangement of origins and destinations (e.g., completion based on spatial locations and proximity) while lacking details about the effects of specific individual and product characteristics. On the other hand, the theories of individual decision-making focus on individual preference as well as product attributes (such as price, quality) and the degree of competition faced by the product as opposed to spatial locations of restaurants. Therefore, travel is not directly addressed in these models. In this study, we combine the two sets of theories to empirically analyze how spatial locations of restaurants, degree of competition, and restaurant characteristics affect traveler's offline restaurant choice behavior.

\section{Data and Empirical Model}

\subsection{Research Setting}

Our research setting is the restaurant choice of consumers who travel by dockless shared bikes (Mobike) in Shanghai, China. Mobike was founded in 2015 with a vision to develop a transportation system by leveraging mobile networks and smart technologies. It was the first cashless, station-free, and the largest (by the number of bikes) shared bicycle operators in the world (Qi, et al., 2018). A user of the dockless bike share service can easily locate and unlock a bike nearby through a smart phone app, and ride it to the destination where he/she parks and locks the bike, making the bike available for other users to use. On April 22, 2016, Mobike launched its official operation in the first city Shanghai followed by rapid expansion into other large cities in China and the international market. By December 2016, Shanghai became the world's largest bike-share city.

Dockless bike sharing provides a flexible, low-cost, and alternative solution for short-distance travel in an urban environment. According to a report released in 2017, about $92 \%$ of the trips shorter than $5 \mathrm{KM}$ are quicker by shared bike plus public transportation; the major purposes of traveling by shared bikes include leisure and exercise, travel to workplaces and schools, travel to restaurants and shops, ride to make public transportation connections. In this study, we focus on the bike trips to restaurants because restaurant is one of the major point of interest (POI) categories in Shanghai, accounting for about $42 \%$ of all POIs. Visiting a restaurant has also been found to be one of the most common short-distance trips to commercial destinations (Millward, et al. 2013). This is consistent with the bike trip patterns in our sample-about 29\% of all trips originated at a restaurant; similar percentage of trips ended at a restaurant.

\subsection{Data}

Our data comes from a number of sources. We obtained a random sample of dockless bike trips in Shanghai from Mobike for the month of August 2016. 
The data set contains 102,361 unique trips. For each trip, the data captures the geolocation of the origin and the destination, the start and the end time of the trip, along with the rider identifier and the bicycle identifier. We also computed the Euclidian distance and the time duration of each trip.

The second source of our data is the POI data in Shanghai. The POI data contains the major point of interests and their geolocations in Shanghai. This data was collected from Gaode map (also known as AutoNavi map) with the latest update in 2015. We further supplemented the POI data to include another major category of the point of interest-residential areas in Shanghai. For each residential area, we extracted the average housing price per square meter from anjuke.com, one of the top online real estate marketplaces in China. We believe that the residential price is a reasonably good indicator of a person's social economic status. Table 1 provides a list of the POI categories as well as their frequency distribution (in percentage) in Shanghai.

Table 1: List of Point of Interest Categories in Shanghai

\begin{tabular}{|l|l|}
\hline POI CATEGORY NAME & PERCENTAGE \\
\hline Dining & $41.73 \%$ \\
\hline Shopping & $33.11 \%$ \\
\hline $\begin{array}{l}\text { Telecommunications } \\
\text { business center/post office }\end{array}$ & $1.27 \%$ \\
\hline Sport activities & $2.30 \%$ \\
\hline Hotel & $0.79 \%$ \\
\hline Tourist attractions & $0.03 \%$ \\
\hline Government building & $0.60 \%$ \\
\hline University/school & $2.56 \%$ \\
\hline Transportation hub/station & $1.79 \%$ \\
\hline Bank & $3.16 \%$ \\
\hline Residential & $12.01 \%$ \\
\hline ADIDAS mall & $0.65 \%$ \\
\hline
\end{tabular}

The third source of our data is a list of restaurants as well as their review data from dianping.com (a Chinese daily deals and local reviews site that is similar to the US-based Yelp). The restaurant review data include consumer ratings (such as stars, taste, environment, and food quality), the average price per person, as well as other restaurant characteristics (including promotion offer, whether the restaurant belongs to a chain) for each restaurant in Shanghai in August 2016. Each restaurant is classified into different categories based on its food offerings, such as Chinese Cuisine, Japanese Cuisine, South East Asian Cuisine, Western Cuisine, Fast Food, Hot Pot,
Seafood, Bakery, Ice Cream, Bar, Drinks, Teahouse, Dessert, etc.

Finally, in order to obtain the bike route distance between the origin and the destination of a trip, we leveraged Baidu Maps service that provides an application program interface (API). The API allows us to extract the shortest bike route distance as well as the time it takes to bike between any pair of locations on Baidu Map. Given that Baidu Map is one of the top map service providers in China as well as the integration of Mobike in Baidu Map's journeyplanning functions, we believe the shortest bike route distance (instead of the Euclidean distance) may better capture the nature of a bike trip.

\subsection{Sample}

Our sampling process comprises of several steps. First, we map each individual trip's origin and destination to the nearest POI based on their geolocations on the map. This allows us to infer an individual's trip purpose and travel behavior because a person who uses dockless bike-sharing service can park the bike as close as possible to the destination. We then filtered our data to include only those trips that originated from a residential area and ended at a restaurant. As we discussed earlier, one of the most common short-distance trips to commercial destinations in an urban setting involves visiting restaurants. Among those trips that ended at a restaurant, about $22 \%$ originated from a residential area. We obtained the average price per square meter for the residential area as discussed above and merged it with the residential-to-restaurant trip data. This set of individual bike trips becomes our target trips/cases.

After that, we created a matching sample of hypothetical trips for each target trip that included all restaurants of the same restaurant type (based on dianping.com restaurant category and the POI restaurant category) within a $1.5 \mathrm{KM}$ radius of the trip origin. These hypothetical trips represented alternative destination choices (restaurants) a bike rider had, but chose not to visit. This matching design is an example of case-control matching (Allison, 2005), in which trips within each match group differ on the choice of a restaurant (the dependent variable). Trips with a chosen restaurant are called cases; trips with an alternative restaurant are called controls. It is noted that a case can be matched to multiple controls because there could be many alternative restaurants of the same type from the origin. Our goal here is to model the determinants of restaurant choice. We further integrated the trip data with the restaurant review data from dianping.dom, the shortest bike route distance 
and the time between each pair of the trip origin and the destination restaurant from Baidu Maps.

Our final data set contains 7,723 observations with 1,406 distinct cases (actual residential-to-restaurant trips). On average, each case has about five matching alternative restaurants (controls) that are of the same restaurant category. The data set is organized in two levels. At the trip level, we capture the trip origin (residential area), the trip distance, the trip duration, and the number of restaurants within a certain radius of the origin. At the restaurant level, we capture the star rating, the service rating, the environment rating, the food rating, the average price per person, the distance and time to the restaurant from the origin, as well as other restaurant characteristics. Our goal is to model the determinants of restaurant choice.

Table 2: Summary Statistics of Data

\begin{tabular}{|c|c|c|c|c|}
\hline Variable & Mean & $\begin{array}{l}\text { Std. } \\
\text { Dev. }\end{array}$ & Min & Max \\
\hline 1. Choice & 0.182 & 0.386 & 0 & 1 \\
\hline $\begin{array}{l}\text { 2. Trip distance (log } \\
\text { meters) }\end{array}$ & 3.247 & 0.281 & 2.24 & 4.32 \\
\hline $\begin{array}{l}\text { 3. Trip duration } \\
\text { (minutes) }\end{array}$ & 17.3 & 19.143 & 3 & 356 \\
\hline $\begin{array}{l}\text { 4. Origin to } \\
\text { restaurant distance } \\
\text { (log meters) }\end{array}$ & 3.12 & 0.239 & 1.51 & 4.32 \\
\hline $\begin{array}{l}\text { 5. Origin to } \\
\text { restaurant duration } \\
\text { (minutes) }\end{array}$ & 7.558 & 4.336 & 0.15 & 104 \\
\hline $\begin{array}{l}\text { 6. Residential price } \\
\text { per sq meter (log } \\
\text { RMB) }\end{array}$ & 4.81 & 0.147 & 3.95 & 5.4 \\
\hline $\begin{array}{l}\text { 7. Number of } \\
\text { alternative } \\
\text { restaurants each trip }\end{array}$ & 12.29 & 8.904 & 1 & 47 \\
\hline $\begin{array}{l}\text { 8. Average price per } \\
\text { person (log RMB) }\end{array}$ & 1.551 & 0.411 & 0 & 3.45 \\
\hline 9. Stars & 3.576 & 0.488 & 0 & 5 \\
\hline $\begin{array}{l}\text { 10. Service rating of } \\
\text { restaurant }\end{array}$ & 7.028 & 0.909 & 0 & 9.3 \\
\hline $\begin{array}{l}\text { 11. Environment } \\
\text { rating of restaurant }\end{array}$ & 7.061 & 0.945 & 0 & 9.3 \\
\hline $\begin{array}{l}12 . \text { Food rating of } \\
\text { restaurant }\end{array}$ & 7.254 & 0.912 & 0 & 9.2 \\
\hline 13. Promotion offer & 0.039 & 0.195 & 0 & 1 \\
\hline 14. Chain restaurant & 0.249 & 0.432 & 0 & 1 \\
\hline $\begin{array}{l}\text { 15. Number of } \\
\text { reviews (log) }\end{array}$ & 2.101 & 0.775 & 0 & 4.48 \\
\hline
\end{tabular}

Table 2 presents the summary statistics of the data set. Subsequent correlation analysis of variables shows that there are high correlations between origin to restaurant distance and origin to restaurant duration; between stars and service rating, environment rating, food rating; between trip distance and trip duration.

\subsection{Variables}

Our data is clustered at the trip level. The primary dependent variable in our analysis is restaurant choice, which is binary and takes a value of " 1 " if an individual visits a restaurant and " 0 " otherwise. Within a cluster (trip), there is one chosen restaurant (i.e., choice $=1$ ) and many other alternative restaurants (i.e., choice $=0$ ). The main trip-level independent variables include trip distance, which measures the proximity of the chosen restaurant to the origin and the number of alternative restaurants for each trip, which measures the competition intensity from the origin of the trip. The main restaurant-level independent variables include the origin to restaurant distance (time), which measures the geospatial distance (travel time) from an origin to a restaurant; the star (service, environment, or food) ratings of a restaurant, which are quality measures of the restaurant; the price per person, which measures the cost of dining at a restaurant. We also control for a number of variables at both the trip level and the restaurant level. These include the residential housing price, which could indicate the social economic status of bike riders, whether a restaurant belongs to a chain, whether a restaurant is offering promotions at the time of the trip, the total number of reviews for the restaurant, etc.

\subsection{Empirical Models}

Given the case-control design, we used conditional logit models in our empirical analysis (Allison, 2005). Equation (1) specifies the complete empirical model (McFadden 1973).

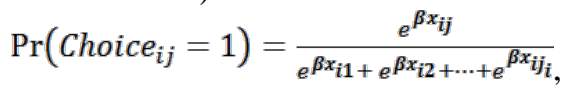

where $\mathbf{x}_{\mathrm{ij}}$ is a vector of restaurant-level input variables (such as average price per person, origin to restaurant distance, star rating) for trip $i$ and restaurant $j, \boldsymbol{\beta}$ is a vector of coefficients. We note that there is no intercept term in the conditional logit model because it cancels out of the model fraction (Allison, 2005). Additionally, Model (1) does not contain cluster level variables (such as competition, trip distance) $\mathbf{x}_{\mathrm{i}}$ because they are the same within each cluster/trip and cannot help predict why a restaurant $j$ rather than another is chosen. In order to capture the effects of these variables, we include interactions between these variables and the restaurant-level variables (Allison, 2005). 


\section{Analysis and Results}

The above conditional logit models can be estimated using PROC LOGISTIC with the STRATA statement in SAS (Allison, 2005). Table 3 presents the results of the conditional logit models.

Table 3: Results of Conditional Logit Model

\begin{tabular}{|c|c|c|c|}
\hline Variables & Model 1 & Model 2 & Model 3 \\
\hline Origin to restaurant distance & $3.197(0.210) * * *$ & $-109.50(6.192) * * *$ & $-109.60(6.222) * * *$ \\
\hline Average price per person & $0.853(0.163) * * *$ & $8.577(3.474) * *$ & $8.268(3.491) * *$ \\
\hline Stars & $-0.006(0.085)$ & $-0.523(1.833)$ & $-0.483(1.854)$ \\
\hline $\begin{array}{l}\text { Origin to restaurant distance * Trip } \\
\text { distance } \\
\text { Average price per person * Trip }\end{array}$ & & $35.692(1.988) * * *$ & $35.727(1.998) * * *$ \\
\hline 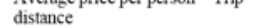 & & $-2.271(1.099) * *$ & $-2.178(1.105) * *$ \\
\hline $\begin{array}{l}\text { Stars }{ }^{*} \text { Trip distance } \\
\text { Origin to restaurant distance * }\end{array}$ & & $0.213(0.589)$ & $0.185(0.595)$ \\
\hline $\begin{array}{l}\text { Competition } \\
\text { Average price per person * }\end{array}$ & & $0.117(0.045)^{\bullet * \bullet}$ & $0.118(0.045)^{* \bullet *}$ \\
\hline Competition & & $-0.049(0.028)^{*}$ & $-0.0495(0.028)^{*}$ \\
\hline Stars * Competition & & $-0.015(0.016)$ & $-0.012(0.017)$ \\
\hline Promotion offer & & & $0.466(0.214) *$ \\
\hline Chain restaurant & & & $0.062(0.114)$ \\
\hline Order out & & & $0.150(0.114)$ \\
\hline Group purchase & & & $-0.131(0.113)$ \\
\hline$-2 \mathrm{LL}$ & 3027.47 & 2103.37 & 2095.79 \\
\hline Number of trips & 1406 & 1406 & 1406 \\
\hline Observations & 7723 & 7723 & 7723 \\
\hline Max re-scaled $R^{2}$ & 0.1159 & 0.4234 & 0.4257 \\
\hline
\end{tabular}

We note several interesting observations and mixed empirical evidence for our hypotheses. Specifically, the coefficient for the star rating of a restaurant is not significant but those for the origin to restaurant distance and the average price per person are both positive and significant in Column 2 of Table 3. This means that star rating has no impact on the choice of the restaurant. This result is a little counter-intuitive, even though previous studies that examine the relationship between online reviews and product sales (e.g., Duan et al., 2008) have documented no significant relationship between the two. Additionally, Luca and Zervas (2016) found that the prevalence of fake or suspicious online restaurant reviews has grown significantly over time on the popular review platform Yelp. It is yet to be seen how the online restaurant ratings affect the consumer's actual decision choice of a restaurant given that the credibility of online reviews has been undermined.

To further investigate how the trip-level distance and competition influence restaurant choice, we included interaction terms of those variables with restaurant-level variables in the regression (Model 2). We see a highly significant negative effect of the trip distance * price interaction (or competition * price interaction), indicating that with increasing travel distance (or competition) there is a decreased preference for a restaurant with a higher price. The main effect of the origin to restaurant distance is significant and negative in Model 2, but the interaction effect of the origin to restaurant distance * trip distance (or origin to restaurant distance * competition) is significant and positive. This indicates that for shorter trip distance, an individual is willing to choose a restaurant that is closer. However, once the trip distance is above a certain level (for example, trip distance $>10^{\wedge} 3.06=1,148$ meters), an individual using dockless bikes does not mind travel additional distance to a restaurant. Finally, Model 3 includes control variables in the regression. We see that there is an increased preference for a restaurant that has a promotion offer. Whether the restaurant belongs to a chain or offers take-out does not seem to affect the individual choice of the restaurant. In all three models, the star rating of the restaurant does not have a significant effect.

\section{Conclusion}

Shared mobility represents an innovative transportation strategy that allows users to make short distance trips on an as-needed basis without the hassle of traditional transportation modes. The recent rise of on-demand ride-sharing systems (Uber, Lyft, Lime, Mobike, Bird, Jump) is having transformative impacts on travelers' attitudes, mobility choices, and behavioral responses to a wide range of daily activities. In this study, we developed a decision choice model, based on the theory of individual decision-making and the spatial interaction model, to examine the restaurant choice behavior of individuals using dockless bikesharing system. We assembled a unique data set that combines the point of interest data, the geospatial information, and the online restaurant review data with the actual bike trips in Shanghai. Our preliminary analysis provides interesting theoretical and practical implications about the consumer buying process when evaluating a restaurant, specifically on how food quality, price, location, and competition would influence the purchase decision.

This study makes a few theoretical contributions. Spatial interaction takes a variety of forms, but usually involves movement of people, goods, or information over physical space that results from a decision-making process (Fotheringham, 2001). The rapid advancement of technology and the digital revolution have significantly empowered consumers in their decisionmaking process. It is therefore important to incorporate key elements from the digital space and examine how those elements influence the spatial flows as well as the choice behavior of consumers in the physical world. In that regard, we hope this study offers insights about some of the underlining factors in the digital 
space that can further calibrate spatial interaction models.

Previous studies in consumer behavior models have found that many factors play a role in a consumer's restaurant choice (Gregory \& Kim, 2004; Njite et al., 2008). Some of the factors (such as consumer demographics, seasonality and day of the week, location) are difficult to control while others (including food quality, price, environment and atmosphere) are relatively easier to change. This study highlights a few important observations to earlier restaurant choice models. Travel distance from an origin to a destination restaurant has different effect on the choice of the restaurant. Shared mobility services provide a flexible and convenient way for consumers to extend travel. Should a consumer decide to travel a long distance, she is more likely to choose a restaurant that is further (among the competitive alternatives). Since physical movement of consumers from one point to another is a necessary condition for many purchase decisions (such as restaurant choice), we believe spatial interaction needs to be modeled in the consumer decision choice process.

There are a few limitations and potential extensions of the study. We assume that an individual would visit a restaurant if it is the nearest POI to her bike drop-off location because we do not have the actual transaction record of the individual at the restaurant. While we have performed additional checks to mitigate the concerns of this assumption. Caution must be exercised when generalizing our findings to other consumer purchasing contexts. We also did not find empirical evidence that the star rating (or quality of that nature) of a restaurant has a significant effect in the consumer's decision choice process. There may be a few reasons for this: star rating is an aggregate metric that involves many dimensions such as price, food quality, service quality, and environment/atmosphere; the users of dockless bike-sharing services do not represent the population. Finally, we did not track and model consecutive decision choices (trip chaining) of individuals, i.e., where do consumers go after they dine at a restaurant. Trip chaining has been an important topic in travel demand and consumer behavior modeling (Primerano, et al., 2008). We call for future research to study these important questions.

\section{References}

[1] Allison, P. 2005. Fixed Effects Regression Models for Longitudinal Data Using SAS. SAS Institute Inc., Cary, NC. [2] Cui, W. 2018. The Effects of Urban Density on the Efficiency of Dockless Bike Sharing System-A Case Study of Beijing, China. Arizona State University Theses and Dissertations.

[3] Davis P. 2006. "Spatial Competition in Retail Markets: Movie Theatres," RAND Journal of Economics, 37(4), pp. 964-982.

[4] Duan, W., Gu, B., and Whinston, A. 2008. "Do Online Reviews Matter? - An Empirical Investigation of Panel Data,” Decision Support Systems, 45(4), pp. 1007-16.

[5] Fotheringham A.S. 1983. "A New Set of SpatialInteraction Models: The Theory of Competing Destinations," Environment and Planning A, 15, pp. 15-36.

[6] Fotheringham A.S. 2001, "Spatial Interaction Models," in International Encyclopedia of the Social \& Behavioral Sciences, eds. N.J. Smelser and P.B. Baltes, pp. 1479414800.

[7] Gerstner, E. 1985. "Do Higher Prices Signal Higher Quality,” Journal of Marketing Research, 22(2), pp. 209-215. [8] Gregory, S., \& Kim, J. 2004. "Restaurant Choice: The Role of Information." Journal of Foodservice Business Research, 7(1), pp. 81-95.

[9] Guldmann J.-M. 1999. “Competing Destinations and Intervening Opportunities Interaction Models of Inter-city Telecommunication Flows,” Papers in Regional Sciences, 78, pp. 179-194.

[10] Hanink D.M. and Stutts, M. 2002. "Spatial Demand for National Battlefield Parks," Annals of Tourism Research, 29(3), pp. 707-719.

[11] Kotler, P. 2000. Marketing Management. Upper Saddle River, New Jersey: Prentice-Hall.

[12] Luca, M. and Zervas, G. 2016. "Fake It Till You Make It: Reputation, Competition, and Yelp Review Fraud," Management Science, 62(12), pp. 3412-3427.

[13] Mas-Colell, A., Whinston, M., and Green, J. R. 1995. Microeconomic Theory. New York: Oxford University Press. [14] McFadden, D. 1973. "Conditional Logit Analysis of Qualitative Choice Behavior," in Frontiers in Econometrics, ed. By P. Zarembka. New York: Academic Press, pp. 105142.

[15] Millward H, Spinney J, and Scott, D. 2013. "Activetransport Walking Behavior: Destinations, Durations, Distances.” Journal of Transport Geography, 28, pp. 101110.

[16] Njite, D., Dunn, G., \& Hyunjung Kim, L. 2008. "Beyond Good Food: What Other Attributes Influence Consumer Preference and Selection of Fine Dining Restaurants?” Journal of Foodservice Business Research, 11(2), pp. 237-266.

[17] Qi, G., Chen, J., and Zhang, Z., "Mobike: A Smart BikeSharing Service Platform," Ivey Publishing, Product Number: 9B18M004, 1/12/2018.

[18] Shaheen, S., Chan, N., Bansal, A., and Cohen, A. 2015. "Shared Mobility: Definitions, Industry Developments, and Early Understanding.” Transportation Sustainability Research Center, Innovative Mobility Research. July 2015.

[19] Shaheen, S., Sperling, D., and Wagner, C. 1998. "Carsharing in Europe and North America: Past, Present and Future,” Transportation Quarterly, 52(3), pp. 35-52.

[20] Simon, H. 1955. "A Behavioral Model of Rational Choice,” The Quarterly Journal of Economics, 69(1), pp. 99118. 
[21] Stouffer, S. A. 1940. "Intervening Opportunities: A Theory Relating to Mobility and Distance," American Sociological Review, 5(6), pp. 845-867.

[22] Smith, T., 1975. "A Choice Theory of Spatial Interaction,” Regional Science and Urban Economics, 5(2), pp. 137-176.
[23] Sproles, G.B., and Kendall, E.L. 1986. “A Methodology for Profiling Consumers’ Decision-making Styles,” Journal of Consumer Affairs, 20 (2), pp 267-279.

[24] Wilson A.G. 1971. "A Family of Spatial Interaction Models and Associated Developments,” Environment and Planning, 3, pp. 1-32. 